\title{
Ankle Distraction Arthroplasty for Ankle Osteoarthritis: A Survival Analysis
}

\author{
Stephen Greenfield ${ }^{1}$, Kelsey M Matta ${ }^{2}$, Thomas H McCoy ${ }^{3}, \mathrm{~S} \mathrm{Robert} \mathrm{Rozbruch}^{4}$, Austin Fragomen ${ }^{5}$
}

\begin{abstract}
Aim: The treatment algorithm for end-stage ankle arthritis is imperfect. Young or active patients are challenging to treat as fusion and replacement carry predictable consequences. Ankle distraction arthroplasty is a less commonly utilized surgical procedure for the treatment of osteoarthritis of the ankle. The purpose of this study was to report intermediate-term survival of ankle distraction and to identify factors associated with earlier time to failure.

Materials and methods: A single-centre, multi-surgeon cohort of 258 cases of ankle arthritis, treated with ankle distraction or ankle distraction with supramalleolar osteotomy (SMO), was identified. Patients were contacted by phone to determine the status of the ankle (natural vs fused/ replaced). Data were collected through chart review. This included patient demographics, medical comorbidities, surgical procedure, and X-ray characteristics including pattern and severity. A Cox regression model was used to determine factors associated with failure during 10 years of follow-up. Risk factors were analysed as hazard ratios (HRs) and 95\% confidence intervals (Cls). Time to failure was illustrated with Kaplan-Meier (KM) curves.

Results: In total, 144 cases were successfully contacted with median follow-up of 4.57 years. In total, $16.7 \%$ of ankles failed (24/144). The 5-year survival was $84 \%$ (95\% Cl: 78-91\%). In adjusted Cox regression, female sex (HR=2.68, $p=0.049)$ and avascular necrosis (AVN) of the talus (HR= $3.77, p=0.041$ ) were significantly associated with failure risk.

Conclusion: Avascular necrosis of the talus and male/female gender differences in survival were found to be significant. Our experience shows that ankle distraction is a valid and effective operation for the treatment of end-stage ankle arthritis.

Clinical significance: This work is clinically significant in that it demonstrates excellent intermediate-term survival data for hinged ankle distraction for treatment of osteoarthritis of the ankle. Additionally, it evaluated patient and disease characteristics allowing improved patient counselling with regard to survival longevity.

Level of evidence: IV cohort study.

Keywords: Ankle arthritis, Ankle distraction arthroplasty, Arthrodiastasis, Avascular necrosis, External fixation.

Strategies in Trauma and Limb Reconstruction (2019): 10.5005/jp-journals-10080-1429
\end{abstract}

\section{INTRODUCTION}

Ankle osteoarthritis is a debilitating condition often due to chronic instability or periarticular trauma affecting a wide age range. Treatment options for ankle arthritis are expanding. Ankle replacement has joined the historic gold standard of fusion as an accepted treatment option for some patients. As the natural history of long-term fusions ${ }^{1}$ and subsequent challenges ${ }^{2}$ has become increasingly evident, improvements in technology and surgeon comfort have increased making ankle replacement gain popularity. However, the latest generation of bone sparing ankle replacements, being championed for younger patients, is less than 5 years old with limited follow-up., It is not clear that younger patients will benefit from replacement surgery. While high-volume arthroplasty centres are now using ankle fusion as a bridge to ankle replacement via fusion "take downs," it is unlikely they will help patients avoid the need for revision arthroplasty in their lifetime given the young age at presentation. Any attempt to devise a treatment algorithm must heavily weigh the age of the patient given the limited durability of current treatment options.

Ankle distraction, or the process of mechanically unloading the joint, is a much less utilized surgical alternative, limited to a few centres around the world. Pain relief is postulated to result from growth of fibrocartilage, resorption of subchondral sclerosis, and ossification of cystic spaces. ${ }^{5,6}$ Various techniques are endorsed in the literature with wide ranges of success reported from largely limited sample size cohorts. ${ }^{7-14}$
${ }^{1}$ Department of Orthopaedic Surgery, Foot and Ankle and Limb Deformity and Reconstructive Surgery, Ortholndy Hospital, Indianapolis, Indiana, USA

${ }^{2}$ Department of Orthopaedic Surgery, Geisinger Commonwealth School of Medicine, Scranton, Pennsylvania, USA

${ }^{3}$ Department of Orthopaedic Surgery, Harvard Medical School, Center for Quantitative Health, Massachusetts General Hospital and Harvard Medical School, Boston, Massachusetts, USA

${ }^{4,5}$ Department of Orthopaedic Surgery, The Hospital for Special Surgery, Weill Medical College of Cornell University, New York, USA

Corresponding Author: Austin Fragomen, Department of Orthopaedic Surgery, The Hospital for Special Surgery, Weill Medical College of Cornell University, New York, USA, Phone: +1 212-606-1550, e-mail: FragomenA@hss.edu

How to cite this article: Greenfield S, Matta KM, McCoy TH, et al. Ankle Distraction Arthroplasty for Ankle Osteoarthritis: A Survival Analysis. Strategies Trauma Limb Reconstr 2019;14(2):65-71.

Source of support: Nil

Conflict of interest: Thomas H McCoy reports unrelated grants from Telefonica Alpha, Brain and Behaviour Research Foundation, and the Broad Institute. Austin Fragomen reports unrelated consultant relationships with Smith + Nephew, Synthes, and NuVasive. S Robert Rozbruch reports unrelated consultant relationships with Smith + Nephew, Stryker, and NuVasive. 
The goal of this study was to evaluate the midterm outcomes of a large cohort of patients treated with ankle distraction arthroplasty. Our primary endpoint was to determine the life span of the native/ natural ankle after ankle distraction, as defined by postponement of ankle fusion, ankle replacement, or repeat distraction. Secondarily, patient characteristics, arthritis patterns, and adjunct surgical procedures were studied to determine prognostic indicators for success.

\section{Materials and Methods}

This retrospective study adheres to ethical principles for medical research and was approved by the hospital institutional review board.

\section{Patient Selection}

Billing records were queried to identify patients from a prospective registry database who were treated for ankle arthritis with ankle distraction arthroplasty between the years of 2001 and 2015. Inclusion criteria included radiographic evidence of ankle arthritis with clinical complaints of debilitating pain, treatment with hinged ankle distraction, and a minimum of $1 \frac{1 / 2}{2}$-year follow-up after date of frame removal. In our experience, a bimodal failure distribution has been seen, and therefore, recent postoperative patients (less than 2 years) were included to avoid omission of these early failures. Patients were excluded if their primary diagnosis was equinus contracture. Patient follow-up was via telephone with each patient being called at least three times, on different days, and at different times to maximize follow-up. Messages and return phone calls were not utilized in order to minimize bias in patient participation. This method of contacting people removed patients from the decisionmaking of whether to call back the research team which should prevent patient outcome from influencing the ability to speak to any given person.

\section{Operative Technique and Frame Assembly}

Frame assembly remained consistent throughout the period of study (Fig. 1). A single tibial ring was fixed with two half pins, perpendicular to the tibial shaft in both the sagittal and coronal planes. Inman's axis, or the axis of rotation for the talocrural joint, was approximated by a temporary llizarov wire inserted from the distal tip of the lateral malleolus to that of the medial malleolus. Two threaded rods with universal hinges were then placed along the axis and fixated to the tibial ring. The position was confirmed on fluoroscopy. Care was taken to avoid binding of the hinges throughout a full range of motion. Medial/lateral olive wires were used for fixation of the calcaneus, taking care to avoid the medial neurovascular structures. A medial-based wire was placed within the talar neck, and depending on surgeon discretion, a second midfoot wire could be used. Multiple adjunct procedures were performed (Table 1). When performed in conjunction with an supramalleolar osteotomy (SMO), a second tibial ring was placed with the middle ring positioned just proximal to the ankle joint. Distraction was kept independent from angular correction at the osteotomy site. Pattern of impingement, prior scars, and surgeon preference dictated joint exposure and preparation method. Microfracture of eburnated bone was performed with a bayonet tip Ilizarov wire. Some patients received a gastroc-soleus release if felt to have a significant equinus contracture. Bone marrow aspirate concentrate (Harvest ${ }^{\oplus}$; Harvest Terumo BCT, Lakewood, CO, USA) was injected after microfracture and distraction and became the standard of care over the last several years performing this procedure. Five millimetres of distraction was applied through the frame: generally, $3 \mathrm{~mm}$ during surgery and $2 \mathrm{~mm}$ on postoperative day 1 after confirming no plantar numbness. Further distraction was applied based on postoperative radiographs until the joint space measured 5-6 $\mathrm{mm}$ on weight-bearing radiographs. The external fixators were worn for 12 weeks.

\section{Clinical Data Mining}

Of the 258 cases of ankle distraction, 144 were successfully contacted by telephone using the above-described protocol. Patients were queried as to status of their ankle (natural vs replaced/fused) and date of subsequent procedures in case of failed distraction. Unknown dates (if performed outside of our institution) were estimated according to the midpoint of the most accurate unit of time known (mid-month, mid-year, etc.). Patient reported outcome scores were not obtained due to a lack of preoperative comparison scores. A chart review was then performed. Age at time of surgery, gender, presence of comorbidities (including diabetes, gout, rheumatoid arthritis, and smoking), and the performance of adjuvant procedures (including extra articular SMO using a hexapod frame, microfracture of sclerotic bone, bone marrow aspirate injection, arthrotomy vs arthroscopy for osteophyte excision, and gastroc-soleus release) were recorded.
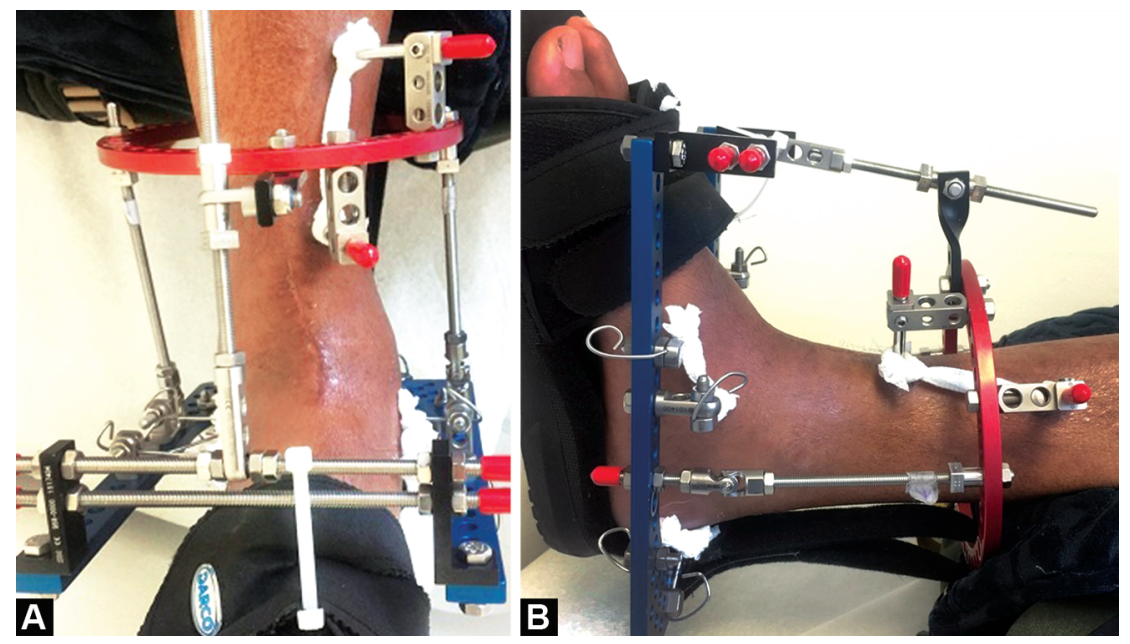

Figs $1 \mathrm{~A}$ and B: (A) Front; (B) Side views of standard hinged frame 
Table 1: Demographics, classification, and procedures for ankle distraction cases

\begin{tabular}{|c|c|c|c|}
\hline & Overall & Survival & Failure \\
\hline$n$ & 144 & 120 & 24 \\
\hline \multicolumn{4}{|l|}{ Demographics } \\
\hline Sex $=M(\%)$ & $70(48.6)$ & $61(50.8)$ & $9(37.5)$ \\
\hline Age [mean (SD)] & $48.9(14.1)$ & $49.3(13.9)$ & $46.8(15.2)$ \\
\hline Laterality = right (\%) & $72(50.0)$ & $58(48.3)$ & $14(58.3)$ \\
\hline $\mathrm{AVN}, n(\%)$ & $10(7.0)$ & $6(5.0)$ & $4(16.7)$ \\
\hline Diabetic, $n(\%)$ & $6(4.3)$ & $5(4.2)$ & $1(4.8)$ \\
\hline Rheumatoid arthritis, $n$ (\%) & $8(5.8)$ & $7(5.9)$ & $1(4.8)$ \\
\hline Tobacco, $n(\%)$ & $15(10.8)$ & $12(10.2)$ & $3(14.3)$ \\
\hline Second opinion, $n(\%)$ & $51(37.0)$ & $46(39.3)$ & $5(23.8)$ \\
\hline \multicolumn{4}{|c|}{ Arthritis pattern and severity on X-ray } \\
\hline \multicolumn{4}{|c|}{ Mortise pattern, $n(\%)$} \\
\hline Neutral & $72(54.5)$ & $62(55.9)$ & $10(47.6)$ \\
\hline Varus & $18(13.6)$ & $14(12.6)$ & $4(19.0)$ \\
\hline Valgus & $17(12.9)$ & $14(12.6)$ & $3(14.3)$ \\
\hline Pantalar & $25(18.9)$ & $21(18.9)$ & $4(19.0)$ \\
\hline \multicolumn{4}{|l|}{ Mortise severity, $n(\%)$} \\
\hline Stages 0-1 & $39(29.5)$ & $33(29.7)$ & $6(28.6)$ \\
\hline Stage 2 & $35(26.5)$ & $27(24.3)$ & $8(38.1)$ \\
\hline Stage 3 & $58(43.9)$ & $51(45.9)$ & $7(33.3)$ \\
\hline \multicolumn{4}{|l|}{ Lateral pattern, $n(\%)$} \\
\hline Anterior $1 / 3$ & $29(22.1)$ & $24(21.8)$ & $5(23.8)$ \\
\hline Anterior $2 / 3$ & $35(26.7)$ & $28(25.5)$ & $7(33.3)$ \\
\hline Concentric & $53(40.5)$ & $45(40.9)$ & $8(38.1)$ \\
\hline Other & $14(10.7)$ & $13(11.8)$ & $1(4.8)$ \\
\hline \multicolumn{4}{|l|}{ Lateral severity, $n(\%)$} \\
\hline Stages 0-1 & $22(16.7)$ & $20(18.0)$ & $2(9.5)$ \\
\hline Stage 2 & $52(39.4)$ & $36(32.4)$ & $16(76.2)$ \\
\hline Stage 3 & $58(43.9)$ & $55(49.5)$ & $3(14.3)$ \\
\hline \multicolumn{4}{|l|}{ Maximum severity, $n$ (\%) } \\
\hline Stages $0-1$ & $18(13.6)$ & $17(15.3)$ & $1(4.8)$ \\
\hline Stage 2 & $43(32.6)$ & $30(27.0)$ & $13(61.9)$ \\
\hline Stage 3 & $71(53.8)$ & $64(57.7)$ & $7(33.3)$ \\
\hline \multicolumn{4}{|l|}{ Adjuvant surgical procedure } \\
\hline $\mathrm{SMO}, n(\%)$ & $38(26.8)$ & $28(23.7)$ & $10(41.7)$ \\
\hline GSR, $n(\%)$ & $43(30.9)$ & $38(33.0)$ & $5(20.8)$ \\
\hline Open arthrotomy, $n$ (\%) & $101(71.1)$ & $85(72.0)$ & $16(66.7)$ \\
\hline $\mathrm{BMAC}, n(\%)$ & $112(80.0)$ & $94(81.0)$ & $18(75.0)$ \\
\hline Microfracture, $n$ (\%) & $35(25.0)$ & $29(25.0)$ & $6(25.0)$ \\
\hline
\end{tabular}

Statistical significance was reserved for the Cox regression analysis

AVN, avascular necrosis; SMO, supramalleolar osteotomy; BMAC, bone marrow aspirate concentrate; GSR, gastrosoleus recession

\section{Radiographic Analyses}

A radiographic review of preoperative images for 132 patients was completed by a single reviewer blinded to patient outcomes. The mortise and lateral X-rays were evaluated for severity and location of disease. The frontal plane location of most severe arthritis was recorded based on involvement of medial gutter (MG), tibiotalar joint (TT), and lateral gutter (LG) as viewed on the mortise X-ray. The patients were then classified according to pattern: "neutral" (TT predominant), "varus" (TT and MG), "valgus" (TT and LG), and "pantalar" (TT, MG, and LG). Similar categorization was based off the lateral X-ray. Patients were categorized into four groups: anterior, central dome and anterior $1 / 3$, concentric, or other. Arthritis severity was graded on a scale of 1-3 according to the ankle osteoarthritis classification as described by Giannini et al. ${ }^{15}$ Severity and location were recorded for both anteroposterior (AP) and lateral X-rays independently (Fig. 2).

\section{Statistical Analyses}

All abstracted variables were summarized to describe the cohort. Continuous variables were summarized with means and standard 

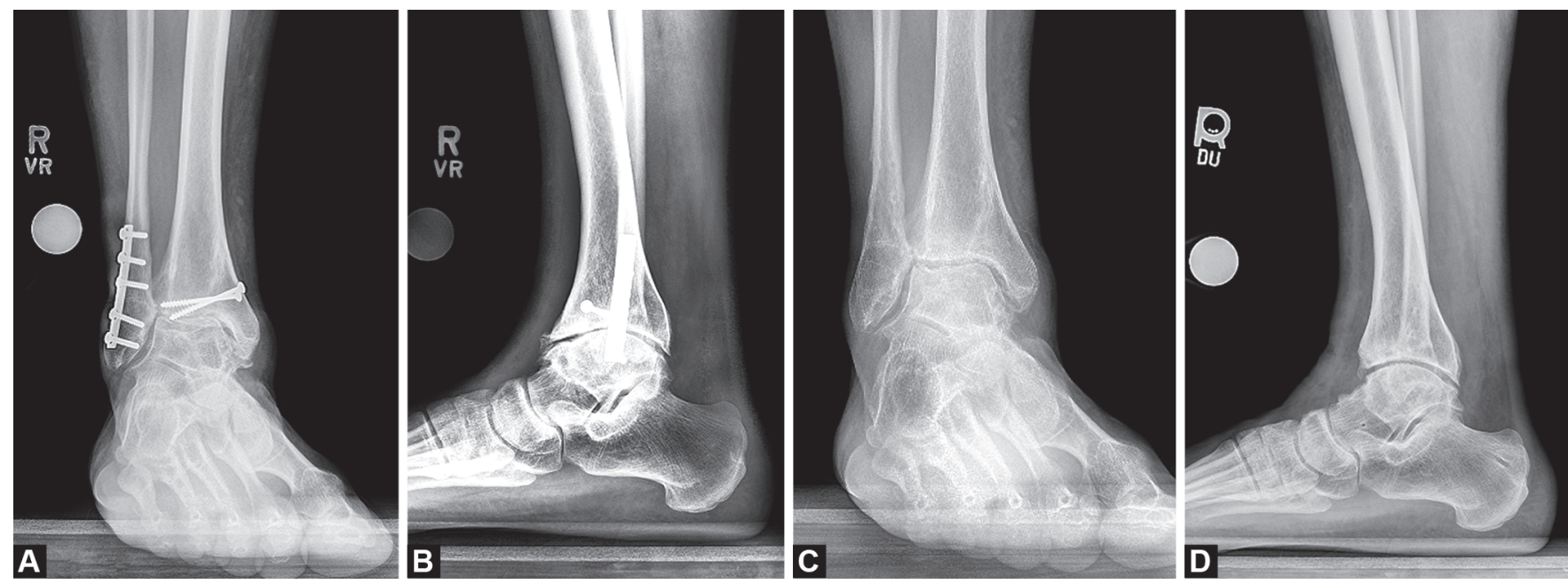

Figs 2A to D: Preoperative and 2-year postoperative X-rays: (A) Preoperative mortise X-ray categorized as "neutral" grade II; (B) Preoperative lateral X-ray categorized as "concentric" grade II; (C) Two-year postoperative mortise X-ray; (D) Two-year postoperative lateral X-ray. There is a modest increase in joint space noted on the postoperative images

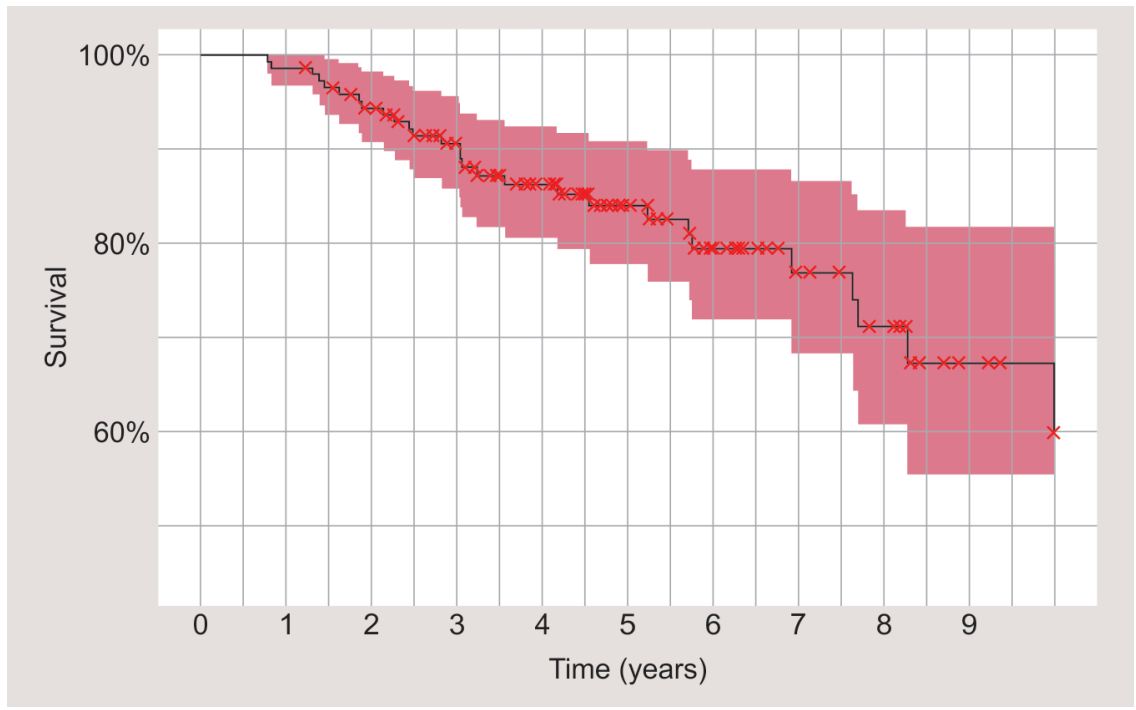

Fig. 3: Kaplan-Meier survival curve censored to 10 years

deviations, and discrete variables were summarized as category counts and percentages. Summaries were calculated both for the full cohort and stratified by ankle survival over the 10-year follow-up period.

A multivariable Cox proportional hazards regression was used to identify associations between patient and surgical variables and time to failure of distracted ankles. Variable inclusion was specified by clinical expertise and included age (years), sex, presence of avascular necrosis (AVN), arthritis severity, AP deformity, and lateral deformity, operative use of gastrosoleus recession (GSR), SMO, or microfracture. Only complete cases were included. The Efron's method was used for ties, and the assumption of proportionality was confirmed using Schoenfeld residuals. Hazard ratios and 95\% confidence intervals (Cls) were calculated. The threshold significance was set at an $\alpha$ level of 0.05 , and a secondary trend value was preselected at an $\alpha$ of 0.1 . All variables which were, or which trended toward, significant were plotted as stratified KM curves showing all complete cases to facilitate interpretation of the Cox regression results. All analysis and plotting was performed using R v3.4.3.

\section{Results}

A total of 144 distracted ankles were identified for inclusion with a median follow-up time of 4.57 years (0.8-14.5 years) resulting in 711 ankle years of follow-up. Patient and operative characteristics, both overall and stratified by outcome, are summarized in Table 1. Over the follow-up period, 24 of 144 (16.7\%) ankles failed (Table 1). The 5- and 10-year survival rates were 84 (95\% Cl: 78-91\%) and $60 \%$ (95\% Cl: 56-82\%), respectively (Fig. 3 and Table 2).

The proportional hazard model included 123 cases as 21 (14.6\%) were removed due to incomplete data. The full model is shown in Table 3. Female sex $(\mathrm{HR}=2.68, p=0.049)$ and presence of radiographic $\mathrm{AVN}$ [hazard ratio $(\mathrm{HR})=3.77, p=0.041$ ] were associated with higher hazard for ankle distraction failure. Supramalleolar osteotomy and intermediate maximum observed 


\begin{tabular}{|c|c|c|c|c|c|c|c|c|c|c|c|}
\hline Time (years) & 0 & 1 & 2 & 3 & 4 & 5 & 6 & 7 & 8 & 9 & 10 \\
\hline At risk (n) & 144 & 142 & 130 & 110 & 86 & 62 & 43 & 29 & 23 & 11 & 9 \\
\hline Failures $(n)$ & 0 & 2 & 6 & 5 & 5 & 2 & 3 & 1 & 2 & 1 & 1 \\
\hline \% Survival & 1.00 & 0.99 & 0.94 & 0.91 & 0.86 & 0.84 & 0.79 & 0.77 & 0.71 & 0.67 & 0.60 \\
\hline
\end{tabular}

Table 3: Cox regression analysis for natural ankle failure within 10 years of distraction arthroplasty

\begin{tabular}{|c|c|c|}
\hline & Adjusted HR & $95 \% \mathrm{Cl}$ \\
\hline Age & 1 & $0.96-1.04$ \\
\hline \multicolumn{3}{|l|}{ Sex } \\
\hline Male & 1 & Ref. \\
\hline Female & $2.68^{*}$ & $1.01-7.15$ \\
\hline \multicolumn{3}{|l|}{ Radiographic AVN } \\
\hline No & 1 & Ref. \\
\hline Yes & $3.77^{*}$ & $1.06-13.40$ \\
\hline \multicolumn{3}{|l|}{ SMO } \\
\hline Distraction & 1 & Ref. \\
\hline Distraction + SMO & $3.11^{\wedge}$ & $0.98-9.89$ \\
\hline \multicolumn{3}{|l|}{ GSR } \\
\hline No & 1 & Ref. \\
\hline Yes & 1.45 & $0.46-4.5$ \\
\hline \multicolumn{3}{|l|}{ Microfracture } \\
\hline No & 1 & Ref. \\
\hline Yes & 0.89 & $0.29-2.77$ \\
\hline \multicolumn{3}{|l|}{ Maximum severity } \\
\hline Stages $0-1$ & 1 & Ref. \\
\hline Stage 2 & $7.69 \wedge$ & $0.75-78.44$ \\
\hline Stage 3 & 1.93 & $0.15-24.10$ \\
\hline \multicolumn{3}{|l|}{ Mortise pattern } \\
\hline Neutral & 1 & Ref. \\
\hline Varus & 2.03 & $0.51-7.98$ \\
\hline Valgus & 1.35 & $0.32-5.63$ \\
\hline Pantalar & 1.14 & $0.31-4.20$ \\
\hline \multicolumn{3}{|l|}{ Lateral pattern } \\
\hline Anterior 1/3 & 1 & Ref. \\
\hline Anterior $2 / 3$ & 1.28 & $0.37-4.39$ \\
\hline Concentric & 0.75 & $0.20-2.84$ \\
\hline Other & 0.6 & $0.06-6.15$ \\
\hline
\end{tabular}

${ }^{*} p<0.05, \wedge p<0.1$

$\mathrm{HR}$, hazard ratios; $\mathrm{Cl}$, confidence intervals; AVN, avascular necrosis; SMO, supramalleolar osteotomy; GSR, gastrosoleus recession

arthritis severity tended toward, but did not reach, significance (Table 3). Surgical treatments such as microfracture or gastroc-soleus release and radiographic characteristics such as pattern of arthritis were not associated with increased or decreased HRs (Fig. 4).

At the time of follow-up, 24 (16.6\%) ankles required repeat operation (10 arthrodesis, 11 total ankle replacement (TAR), and 3 repeat distraction arthroplasty). These patients reported no complication of their secondary surgery.

\section{Discussion}

Although ankle distraction arthroplasty is not a new technique, it is infrequently utilized, and research on this procedure is sparse.
This study provides significant additional evidence that ankle distraction is a viable joint sparing procedure for the treatment of ankle arthritis with $84 \%$ survival at 5 years. This represents an improvement in intermediate-term results than previously published. Existing literature demonstrates a range of survival data. An early series out of the Netherlands showed $73 \%$ survival at 7 years although patient numbers were limited (27 patients). ${ }^{16}$ In a significantly larger series of 111 patients, ${ }^{17}$ the authors demonstrated $56 \%$ survival with $17 \%$ failing within 2 years and $37 \%$ within 5 years. A randomized controlled trial comparing fixed vs hinged ankle distraction at an average of 8.3 years for 29 patients demonstrated a $44 \%$ conversion rate to total ankle or fusion. ${ }^{12}$ Interestingly, patients treated with hinged distraction had improved ankle osteoarthritis scale scores at the 2 -year time point ${ }^{12}$ while fixed distraction patients had improved scores at intermediate-term follow-up. ${ }^{18}$ Differences in survival results between these studies are hard to attribute to any one factor given the inherent differences in indications, techniques, and postoperative protocols as there is no consensus on any one of these features. Our evolving multimodal approach to distraction arthroplasty, including the addition of microfracture and bone marrow aspirate concentrate, could be confounding to the experimental process but, likewise, may be responsible for our success. Providing optimal and treatment customized to each patient is a shortcoming of clinical research but makes the results representative of the decision process and entire surgical complement being practiced.

A Cox proportional hazard model was used to determine demographic, clinical, and adjunct procedures that may impact survival. Both sex and radiographic evidence of talus AVN proved to be predictors of treatment outcome. Male patients demonstrated improved longevity of their native joint when compared with their female counterparts. Marijnissen et al. demonstrated a drastic difference in success between men and women. In their report, $30 \%$ of women converted to fusion or replacement by 2 years, a rate higher than men's failure at 11 years. ${ }^{17}$ They do not offer explanation for this observation but reference a gender bias found in prior research focusing on hip distraction in setting of juvenile AVN..$^{19}$ The durability difference seen in our study is significantly less drastic with 5 -year survival at 88 and $80 \%$ for men and women, respectively. The difference in success demonstrated between these studies is noteworthy. Regardless of the cause, we felt this subtle difference was not clinically significant to recommend against distraction for female patients but does provide basis for further patient counselling.

The presence of radiographic AVN was a predictor of worse outcome with 5 -year survival of $70 \%$ compared with $88 \%$ of the non-AVN cohort. Assuming persistent pain drives need for revision, we conclude that pain relief is less in this cohort. Several studies have proposed mechanisms of pain reduction for ankle distraction. Intema et al. studied radiographic consequences of joint distraction and correlated them with improved pain control. ${ }^{6}$ Radiographic density (Hounsfield units) was compared on preoperative and postoperative computed tomography (CT) scans through computer- 

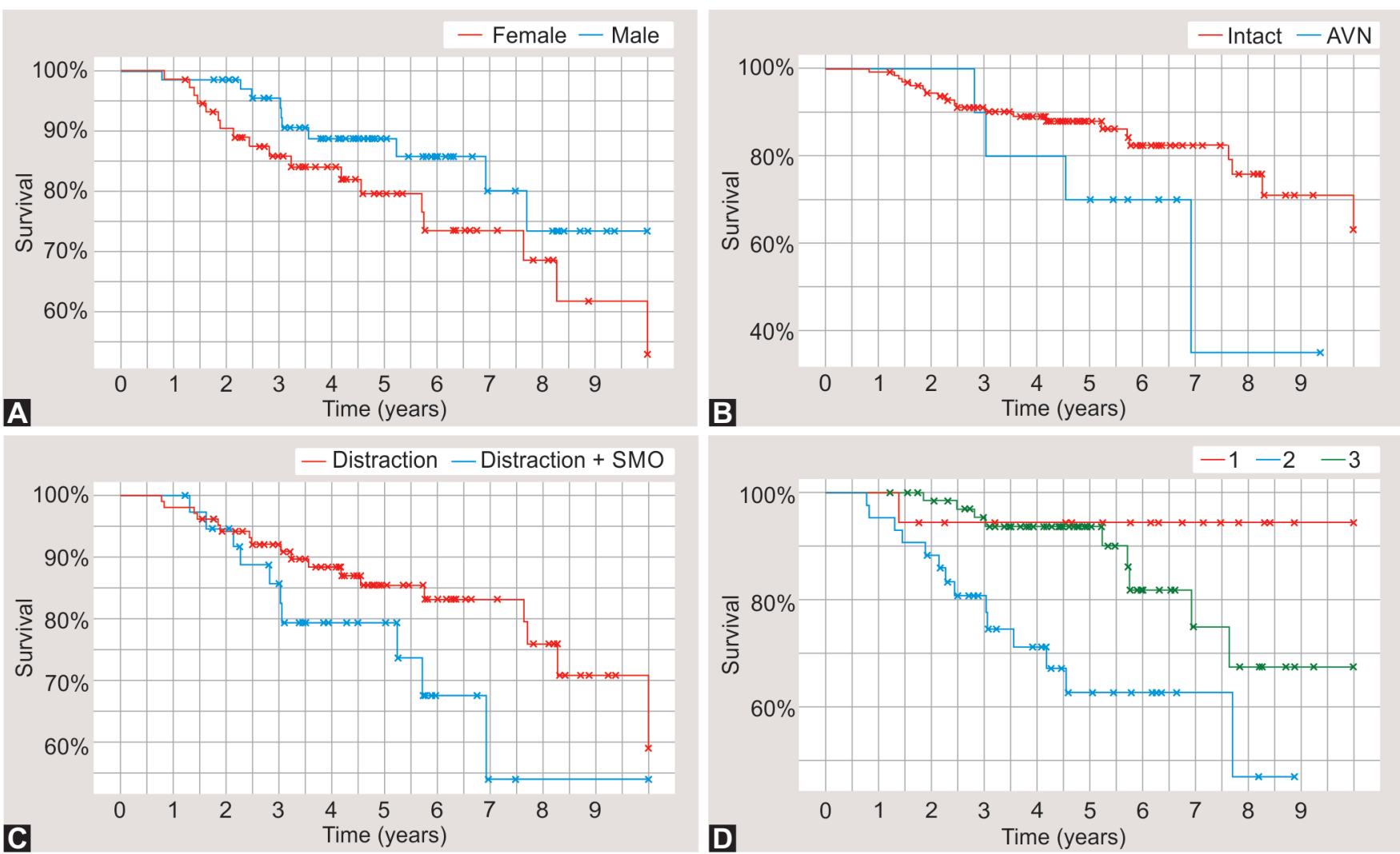

Figs 4A to D: Kaplan-Meier curve of 10-year ankle survival stratified by (A) Sex ( $p=0.049)$; (B) Radiographic AVN status ( $p=0.041)$; (C) Deformity requiring SMO $(p=0.054)$; (D) Maximum arthritis severity on X-ray $(p=0.085)$

assisted overlap techniques. They observed a decrease in density of sclerotic regions and an increase in density in cystic regions when comparing postoperative to preoperative CT scans. Clinical improvement was found to correlate with cyst ossification. Other work demonstrated that $61 \%$ of patients experienced a statistically significant increase in radiographic joint space on weight-bearing films. ${ }^{14}$ While they did not report on survival of this subgroup relative to those that did not demonstrate increased radiographic joint space, they postulate this new fibrocartilaginous buffer contributed to pain relief. In the setting of AVN, we suspect there is insufficient interosseous perfusion to allow for bone remodelling and delivery of fibrocartilaginous progenitor cells to the articular surface. Thus, both mechanisms for pain relief are jeopardized in AVN; however, most patients with AVN responded positively to the distraction arthroplasty (DA) surgery as seen in our data.

We found a difference in survival that was not statistically significant when comparing patients with neutral joint alignment treated with distraction against those who were treated with distraction and SMO for joint malalignment. Supramalleolar osteotomies were performed to restore natural joint angles and minimize pathologic shear forces across the plafond. The goal was to realign, postpone fusion or replacement, and in some cases, correct deformity in order to allow for future ankle replacement. The KM survival curve demonstrated 5-year survival for a neutral ankle to be $86 \%$ compared with $79 \%$ for SMO patients. However, when comparing these groups, the supramalleolar group had more severe radiographic arthritis and more frequently had involvement of either joint gutter (most SMOs were performed for correction of coronal malalignment). Despite the modest decrease in survival, we feel this is still a valuable option for these patients who have more complex pathology. Our model failed to demonstrate a clear clinical explanation for survival as a function of arthritis severity. Patients with grades I and III experienced improved survival over grade II. Given grade III is characterized by complete obliteration of the joint space and advanced sclerosis with cyst formation. It is possible that pain control through remodelling of epiphyseal bone (resorption of sclerosis and ossification of cysts) leads to significantly more relief than in the less advanced grade II or that greater functional disability preoperatively seen in grade III was predictive of less postoperative pain. ${ }^{17}$ No consistent difference in survival as a function of arthritis pattern was found.

This present study has several limitations. In order to accumulate large numbers of patients, this work represents the evolution of ankle distraction within our practice. During the course of the study, there were changes in the philosophy toward appropriate distraction gap, use of concentrated bone marrow aspirate, and joint preparation techniques among other things. Although a surgical technique was largely consistent between surgeons, technique evolution occurred at different rates and thus we cannot stratify by time in order to improve uniformity. We were also not able to comment on the impact of body mass index on survival (due to a lack of data), which has been identified as a risk factor for early failure. ${ }^{14}$ Patient reported outcome scores comparing before and after results would have greatly strengthened this study, but we felt that a postoperative score without a preoperative comparison was not meaningful and was, therefore, not obtained during the follow-up call. The research team was limited in our ability to contact patients without inviting biased call backs from those patients. The concern was that a patient who was performing poorly after the procedure would be less inclined to return the phone call. By "cold 
calling" everyone, interviewing only those people who answered the phone and not leaving messages, we attempted to mitigate this bias. Many of our patients were younger and from out of state making contacting them by phone formidable. Email was not used for the same bias concerns. Any assumption that the 114 patients we were unable to reach by phone all failed treatment is unfounded.

\section{Conclusion}

Ankle distraction is an important alternative to fusion and replacement in the treatment of post-traumatic ankle osteoarthritis. Both males and females can benefit from this surgery with different survival expectations. Distraction arthroplasty can be used in cases of ankle arthritis associated with talar AVN with the understanding that the results may be inferior to non-necrotic cases. Given the challenges with arthritis treatment in young patients as well as the growing numbers of middle-aged and elderly patients who desire joint preservation techniques, we feel ankle distraction arthroplasty is an important treatment that can prolong the need for terminal procedures such as fusion and replacement. Patients who failed distraction and went on to have these other procedures reported no adverse events supporting the contention that ankle distraction does not "burn bridges."

\section{Clinical Significance}

This work is clinically significance in that it demonstrates excellent intermediate-term survival data for hinged ankle distraction for treatment of osteoarthritis of the ankle. A better understanding of patient and disease characteristics will allow for improved patient counselling with regard to survival longevity.

\section{Manufacturer Name}

The product used in this study was the "RAD Frame" from Stryker (Mahwah, NJ, USA).

\section{ACKNOWLEDgments}

No sponsors had a role in study design, writing of the report, or data collection, analysis, or interpretation. The first and senior authors had full access to all data and made the decision to submit for publication. The study was approved by the institutional review board.

\section{References}

1. Coester LM, Saltzman CL, Leupold J, et al. Long-term results following ankle arthrodesis for post-traumatic arthritis. J Bone Jt Surg Am 2001;83(2):219. DOI: 10.2106/00004623-200102000-00009. http:// www.ejbjs.org/cgi/content/abstract/83/2/219\%5Cnhttp://www. ejbjs.org/cgi/reprint/83/2/219.pdf.

2. Bugbee WD, Khanna G, Cavallo M, et al. Bipolar fresh osteochondral allografting of the tibiotalar joint. J Bone Joint Surg Am 2013;95(5): 426-432. DOI: 10.2106/JBJS.L.00165.

3. Daniels TR, Younger AS, Penner M, et al. Intermediate-term results of total ankle replacement and ankle arthrodesis. J Bone Joint Surg Am 2014;96(2):135-142. DOI: 10.2106/JBJS.L.01597.
4. Penner $M$, Davis WH, Wing $K$, et al. The infinity total ankle system: early clinical results with 2- to 4-year follow-up. Foot Ankle Spec 2018;11(4):193864001877760. DOI: 10.1177/1938640018777601.

5. Marijnissen ACA, Van Roermund PM, Van Melkebeek J, et al. Clinical benefit of joint distraction in the treatment of severe osteoarthritis of the ankle: proof of concept in an open prospective study and in a randomized controlled study. Arthritis Rheum 2002;46(11): 2893-2902. DOI: 10.1002/art.10612.

6. Intema F, Thomas TP, Anderson DD, et al. Subchondral bone remodeling is related to clinical improvement after joint distraction in the treatment of ankle osteoarthritis. Osteoarthr Cartil 2011;19(6): 668-675. DOI: 10.1016/j.joca.2011.02.005.

7. van Roermund PM, Marijnissen ACA, FPJG Lafeber. Joint distraction as an alternative for the treatment of osteoarthritis. Foot Ankle Clin 2002;7(3):515-527. DOI: 10.1016/S1083-7515(02)00027-X.

8. van Valburg A, van Roermund P, Lammens J, et al. Can llizarov joint distraction delay the need for an arthrodesis of the ankle? A preliminary report. J Bone Jt Surg Br 1995;77(5):720-725. DOI: 10.1302/0301-620X.77B5.7559696. http://www.ncbi.nlm.nih.gov/ entrez/query.fcgi?cmd=Retrieve \&db=PubMed\&dopt=Citation\&l ist_uids $=7559696$.

9. Zhang K, Jiang Y, Du J, et al. Comparison of distraction arthroplasty alone versus combined with arthroscopic microfracture in treatment of post-traumatic ankle arthritis. J Orthop Surg Res 2017;12(1):45. DOI: 10.1186/s13018-017-0546-7.

10. Barg A, Amendola A, Beaman DN, et al. Ankle joint distraction arthroplasty. Foot Ankle Clin 2013;18(3):459-470. DOI: 10.1016/j. fcl.2013.06.005.

11. Bernstein M, Reidler J, Fragomen A, et al. Ankle distraction arthroplasty. J Am Acad Orthop Surg 2017;25(2):89-99. DOI: 10.5435/ JAAOS-D-14-00077.

12. Saltzman $\mathrm{CL}$, Hillis $\mathrm{SL}$, Stolley MP, et al. Motion versus fixed distraction of the joint in the treatment of ankle osteoarthritis: a prospective randomized controlled trial. J Bone Joint Surg Am 2012;94(11):961970. DOI: 10.2106/JBJS.K.00018.

13. Zhao HM, Liang XJ, Li Y, et al. Supramalleolar osteotomy with distraction arthroplasty in treatment of Varus ankle osteoarthritis with large talar tilt angle: a case report and literature review. J Foot Ankle Surg 2017;56(5):1125-1128. DOI: 10.1053/j.jfas.2017.04.022.

14. Zhao H, Qu W, Li Y, et al. Functional analysis of distraction arthroplasty in the treatment of ankle osteoarthritis. J Orthop Surg Res 2017;12(1):18. DOI: 10.1186/s13018-017-0519-x.

15. Giannini S, Buda R, Faldini C, et al. The treatment of severe posttraumatic arthritis of the ankle joint. J Bone Jt Surg - Ser A 2007;89(Suppl. 3):15-28. DOI: 10.2106/JBJS.G.00544.

16. Ploegmakers JJW, van Roermund PM, van Melkebeek J, et al. Prolonged clinical benefit from joint distraction in the treatment of ankle osteoarthritis. Osteoarthr Cartil 2005;13(7):582-588. DOI: 10.1016/j.joca.2005.03.002.

17. Marijnissen ACA, Hoekstra MCL, Pré BC, et al. Patient characteristics as predictors of clinical outcome of distraction in treatment of severe ankle osteoarthritis. J Orthop Res 2014;32(1):96-101. DOI: 10.1002/ jor.22475.

18. Nguyen MP, Pedersen DR, Gao Y, et al. Intermediate-term follow-up after ankle distraction for treatment of end-stage osteoarthritis. J Bone Joint Surg Am 2015;97(7):590-596. DOI: 10.2106/JBJS.N.00901.

19. Gomez JA, Matsumoto $\mathrm{H}$, Roye DP, et al. Articulated hip distraction: a treatment option for femoral head avascular necrosis in adolescence. J Pediatr Orthop 2009;29(2):163-169. DOI: 10.1097/BPO.0b013e31819 903b9. 